\title{
Study of blood pressure to height ratio and its relation with standard BP percentile charts to detect the prehypertension in age group 12 to 15 yrs
}

\author{
Kurane AB ${ }^{1}$, Kurane IA ${ }^{2}$, N S Magdum ${ }^{3}$, S Sumana ${ }^{4}$ \\ ${ }^{1}$ Dr. A B Kurane, Professor and HOD, Department of Pediatrics, ${ }^{2}$ Dr. Mrs. I A Kurane, Assistant Professor, Department \\ of Physiology, ${ }^{3}$ Dr. N S Magdum, Post Graduate student, Department of Pediatrics, ${ }^{4}$ Dr. S Sumana, Post Graduate \\ student, Department of Pediatrics. All are affiliated with Dr D Y Patil Medical College and Research Institute, Kolhapur, \\ India
}

Address for Correspondence: Dr. A B Kurane, Email: drkuraneanil@yahoo.com

\begin{abstract}
Introduction: The prevalence of adolescent (pre) hypertension, even in developing countries, is rising steeply, probably due to urbanization and the positive energy balance - typified by excess weight gain - that comes with it. Primary prevention of one of the leading health problems, i.e. hypertension and ischemic heart disease in adults necessitates a scientific evaluation of the predictors in children. Currently, blood pressure percentile in childhood is assessed according to age, gender, height. Aim \& Objectives: To study blood pressure to height ratio and its relation with standard BP percentile charts to detect (pre) hypertension. Methodology: BP percentile was calculated for 2000 adolescents. Receiver operating characteristic (ROC) curve analyses were performed to calculate sensitivity and specificity of BP/height ratios as diagnostic tests for elevated BP (90\%). Correlation analysis was performed between BP percentile and BP/height ratios. Result: In our study average age was $13.1 \pm 1.2$ years. SBP/height ratio was $\geq 0.7607$ and $\geq 0.7795$ in boys and girls respectively. And DBP/height ratio was $\geq 0.4513$ and $\geq 0.46$ in boys and girls respectively. The ratios strongly correlate with the BP percentile charts with high sensitivity and specificity. Conclusion: The BP/height ratios correlate with the corresponding standard BP percentiles charts in both genders. It may eliminate the under-diagnosis of adolescent (pre)hypertension and hypertension, and in turn, may help to reduce morbidity and mortality resulting from its sequelae.
\end{abstract}

Keywords: Adolescent, Hypertension, Systolic BP(SBP), Diastolic BP(DBP).

\section{Introduction}

Hypertension is a global health problem and it is well documented that increased blood pressure becomes established in childhood [1,2]. Hypertension in children and adolescents is defined as systolic BP (SBP) and /or diastolic BP (DBP) above the $95^{\text {th }}$ percentile. BP between the $90^{\text {th }}$ and $95^{\text {th }}$ percentile is designated "high normal" or pre- hypertensive [2].

The prevalence of prehypertension and hypertension increases progressively with increasing body mass index (BMI) and hypertension is detectable in over 30\% of overweight children with BMI above 95th percentile [3]. Hypertension in childhood and adolescence has been a proven risk factor for hypertension in adult [4, 5].

Manuscript received: $17^{\text {th }}$ Nov 2015

Reviewed: $24^{\text {th }}$ Nov 2015

Author Corrected: $8^{\text {th }}$ Dec 2015

Accepted for Publication: $19^{\text {th }}$ Dec 2015
Currently the available data to quantify hypertension in children is in the form of a table reference matched by age, gender, and height percentile that identifies the BP as $50^{\text {th }}, 90^{\text {th }}, 95^{\text {th }}$ and $99^{\text {th }}$ percentile [2].

The criteria for diagnosis of pre hypertension and hypertension in children and adolescents can be complex and challenging to apply in clinical practice because of age, gender and height specific reference values [6]. Both pre hypertension and hypertension though prevalent and increasing in incidence remain widely undiagnosed.

Lu et al [6] reported that blood pressure to height ratios were both feasible and accurate as diagnostic tool for (pre) hypertension and hypertension in Han adolescents of China, and proposed optimal thresholds for SBP to 
height ratio (SBPHR) and DBP to height ratio (DBPHR) for the said population. This study recognizes the racial differences in adolescent hypertension $[7,8]$.

These studies have recommended determining the sensitivity and specificity of these indices in various populations. In order to validate the $\mathrm{BP} /$ Height ratios, similar studies are needed to be carried out. This has formed the basis of our study.

In our study we have tested that simple BP/ Height ratios can be used as accurate clinical tests to identify children and adolescents at risk for pre hypertension and hypertension. We also report the co relation of BP/ height ratios with commonly used BP percentile charts.

\section{Methodology}

Study Population: A cohort study of 2000 children was conducted in Dr. D. Y. Patil Hospital. The data used in this study is collected from private schools in Kolhapur city. The study sample consist of $2000(1,398$ boys and 602 girls) school children, in the age group of 12-15 years. A sample size was obtained after detailed discussion with statistician.

\section{Inclusion Criteria}

All school going boys and girls between age $12-15$ yrs.

\section{Exclusion Criteria}

Children with history of known secondary hypertension were excluded from study.

The study was approved by Research and Ethical committee of Dr D Y Patil Medical College and Research Institute. Permission from the concerned authority of schools was obtained to conduct the study among school children. After detailed explanation of the objectives and protocols of the study, informed consents were obtained from parents. Parents were asked if there was any history of hypertension due to secondary causes in children and if child is on any antihypertensive medications and such children were excluded from the study.

Procedure and Measurements: The following parameters were taken. Age, sex, weight, height, systolic and diastolic blood pressure. Age in completed years was recorded as per school admission registers.

Measurements were made by a single person and same equipment was used to obtain accurate measurement and to increase the sensitivity of the results. Weight was recorded using spring balance (bathroom scale) calibrated to $0.5 \mathrm{~kg}$ accuracy.

The height was measured by making the child to stand upright, barefoot on the ground with heels, head in Frankfurt plane. The height was measured using sliding stadiometer (Johnson and Johnson) with an accuracy of $1 \mathrm{~mm}$.

Diamond mercury manometer was used to record blood pressure, with a set of different sized cuffs as per the recommendation given by the fourth report on the diagnosis, evaluation and treatment of high blood pressure in children28. The cuff bladder was wide enough to cover at least $2 / 3$ of arm and long enough to encircle arm completely. Auscultatory method was used and the 1st and 5th Korotkoff's sounds were taken as indicative of the systolic and diastolic blood pressure respectively. Systolic blood pressure (SBP) and diastolic blood pressure (DBP) were reported to be measured by a standard protocol used by NHANES 8 . Blood pressure was recorded 3 times with 2 min interval between each. All recordings were done by a single individual to eliminate observer subjective bias. Before recording blood pressure, the procedure was explained to children and sufficient time was given to allay anxiety and fear. Blood pressure was measured in supine position. In children where a higher range of blood pressure was observed, the factors like anxiety and fear were removed and re-recorded after one hour. Average of 3 BP readings was taken.

Statistical Analysis: Correlation coefficient and simple linear regression analysis was done for predicting blood pressure (SBP and DBP) separately for age, sex, weight and height. BP percentiles were calculated according to the standard formula provided in the following reference manuscript.

Statistical Analysis: Receiver-operating characteristic (ROC) curve analyses were performed to calculate sensitivity and specificity of SBP/height and $\mathrm{DBP} /$ height ratios as diagnostic tests for elevated, that is, $>90$ th percentile of SBP and DBP, respectively.

Correlation analysis was performed between SBP percentile and SBP/height ratio. Similar correlation was studied for DBP percentiles and DBP/height ratio. ROC analysis and curve generation were performed using Analyse-it Method Evaluation edition. For data analysis and interpretation we used SPSS v.19. 


\section{Results}

This study included 2000 children in the age group 12 to 15 years. Participants consisted of 1398 boys aged $13.1 \pm 1.2$ years old and 602 girls aged $13.0 \pm 1.0$ years old. The proportion of BP above $90^{\text {th }}$ percentile in all boys and girls was $2.57 \%$ (36 out of 1398 boys) and $3.98 \%$ (24 out of 602 girls) respectively.

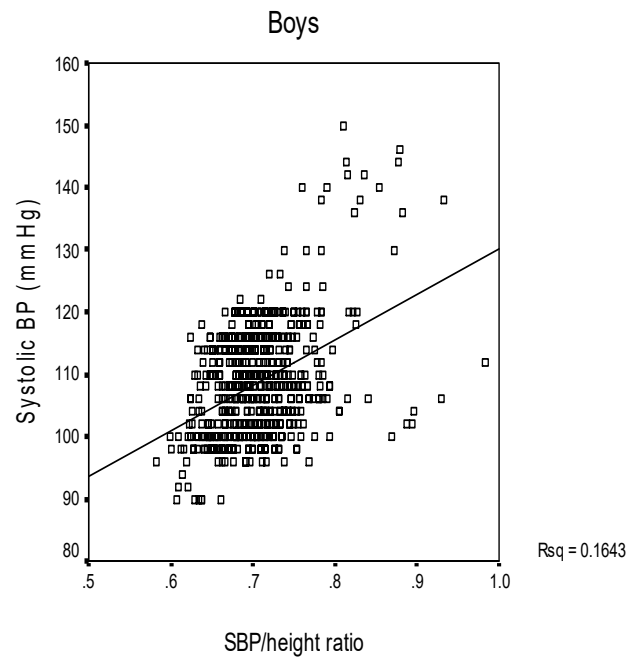

Figure 1(a)

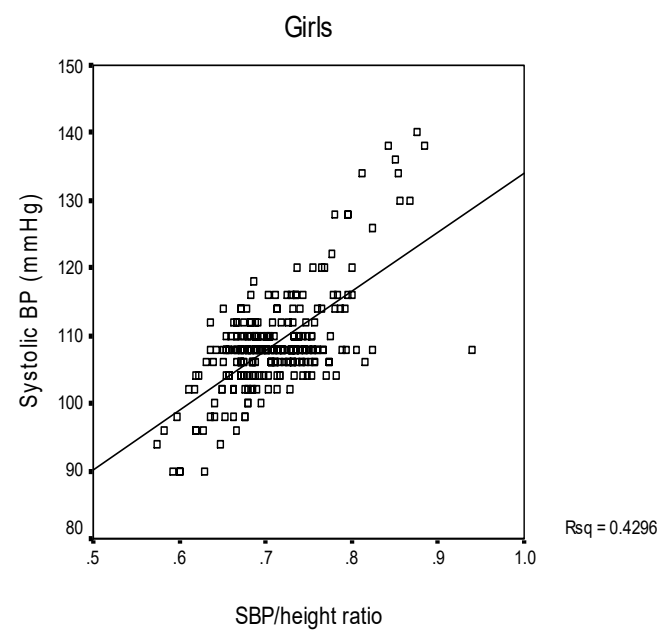

Figure 1(b)

SBP / Height ratio strongly correlated with SBP percentiles in both boys (Figure 1(a); $P<0.001, \mathrm{R}^{2}=0.1643$ ) and girls ( Figure $\left.1(\mathrm{~b}) ; P<0.001, \mathrm{R}^{2}=0.1643\right)$.
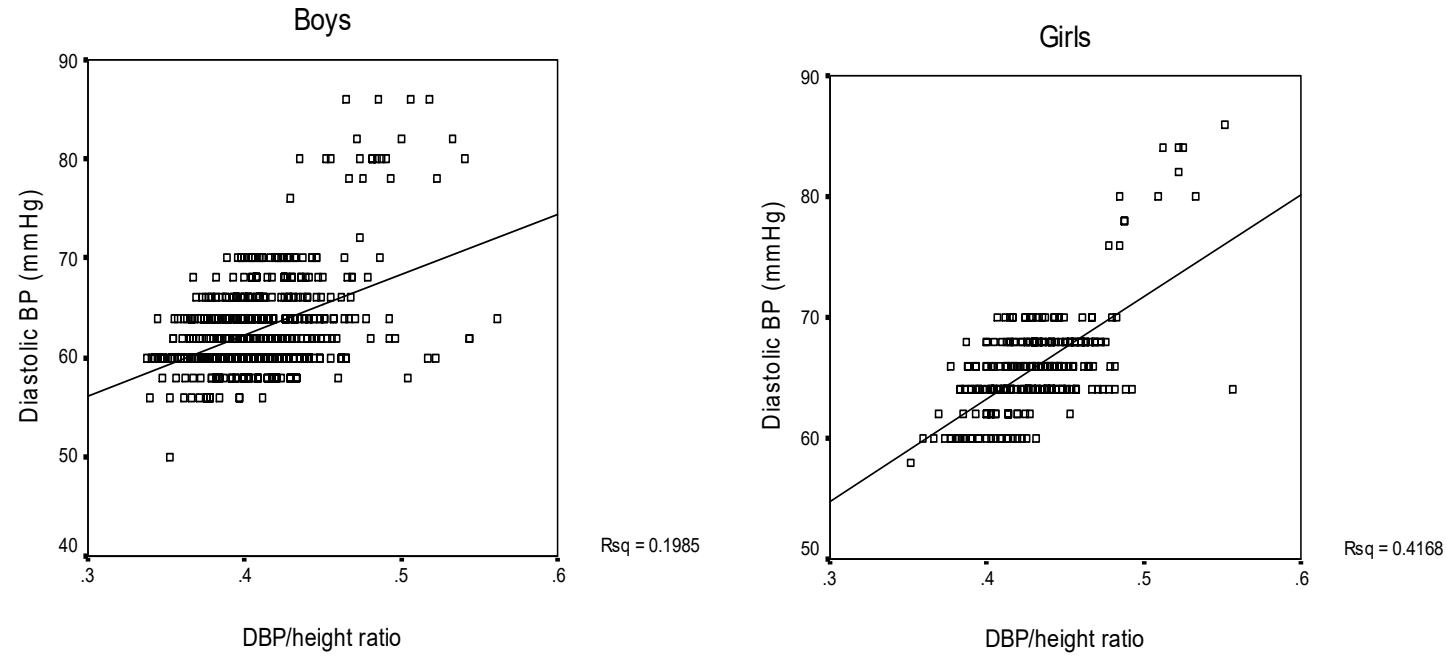

Figure 2(a) Figure 2(b)

Similar results were obtained for DBP/ Height ratio and DBP percentiles : boys (Figure $2(\mathrm{a}) ; P<0.001, \mathrm{R}^{2}=0.1985$ )and girls (Figure 2(b); $P, 0.001, \mathrm{R}^{2}=0.1985$ ).

ROC analysis showed a very steep progression of sensitivity and specificity above certain cut off values. The cut off values of elevated SBP/Height ratios in boys and girls was $\geq 0.74$ and $\geq 0.78$ respectively.

The cut off values for elevated DBP/Height ratios was $\geq 0.44$ in boys and $\geq 0.49$ in girls. Above the cut off values of the ratios the sensitivity and specificity of detection of elevated $\mathrm{BP}\left(>90^{\text {th }}\right.$ percentile $)$ was very high. 


\section{Discussion}

Hypertension in the pediatric and adolescent population may remain undiagnosed, although the BP charting for age, gender and height are readily available. They are generally not used in a busy pediatric practice, so the diagnosis of hypertension may be missed in some children.

This study may provide a simplified diagnostic test for primary assessment of BP and for detecting children \& adolescent in need of further follow up for diagnosis of pre-hypertension \& hypertension.

In this study we have shown that $\mathrm{BP} /$ Height ratios are simple $\&$ accurate method of diagnosing elevated blood pressure. Our findings are in complete agreement with preceding studies on this matter.

The author in respective studies have clearly demonstrated the feasibility and accuracy of BP/Height \& DBP/Height ratios in there cohort of Chinese, Italian and Nigerian adolescents, in our study the cut off for $\mathrm{SBP} /$ Height ratio for both boys and girls in $\geq 0.74$ and $\geq 0.78$ respectively[ $[6,9,10]$.

The DBP/Height ratio in boys \& girls are $\geq 0.44$ and $\geq 0.49$ respectively. The sensitivities and specificities of these ratios are from $84.4 \%$ to $98 \%$ in identifying elevated BP above the $90^{\text {th }}$ percentile. Present study shows both $\mathrm{SBP} /$ height ratio and $\mathrm{DBP} /$ height ratio correlates with the corresponding BP percentile charts. $\mathrm{p}$ value of present study is $<0.001$ it states that values of $\mathrm{SBP} /$ height ratio and $\mathrm{DBP} /$ height ratio is statistically significant. Similarly $\mathrm{Lu}$ et al, Chukwunonso ECC Ejike, Ovidiu Galescu et al shows P value $<0.001$. It shows that present study correlates with the above studies[6,9,10]. This study may be limited by some factors. First, the not-too-large sample size for this study may imply a debatably reduced statistical power of entire analysis. Though a larger population may yield different threshold values, the differences may not be significant. These results therefore call for a very much larger national (or even regional) study aimed at determining cut off values for SBPHR and DBPHR in India.

\section{Conclusion}

$\mathrm{BP} /$ Height ratio is a simple, excellent screening and diagnostic tool with high sensitivity and specificity to detect (pre)hypertension. The BP/Height ratios also corelate with the corresponding standard BP percentile charts in both genders. The $\mathrm{BP} /$ Height ratio can be easily used in routine medical care of children to detect prehypertension.

It is suggested that the use of this simple, inexpensive and accurate indices should be standardized into screening programs for diagnosis of elevated BP in the pediatric age group. It would be appreciated by non medical professionals as well.

It may also eliminate the under- diagnosis of adolescent (pre)hypertension and in turn, help in the early management of cases, and ultimately a reduction in the morbidity and mortality arising from its sequale.

Ethical approval: Obtained from Institutional Ethics Committee (Human Studies)

Conflict of Interest: The authors declare no conflict of interest

Funding: Nil

\section{References}

1. Galescu O, George M, Basetty S, Predescu I, Mongia A, Ten S, Bhangoo A. Blood Pressure over Height Ratios: Simple and Accurate Method of Detecting Elevated Blood Pressure in Children. Int $J$ Pediatr. 2012;2012:253497. doi: 10.1155/2012/253497. Epub 2012 Apr 8

2. Chobanian A V, Bakris G S, Black H R, Cushman W C, Green L A, Jones D W et al., Seventh report of the Joint National Committee on Prevention, Detection, Evaluation, and Treatment of High Blood Pressure Hypertension. 2003; 42: 1206-1252.

3. Sorof JM, Lai D, Turner J, Poffenbarger T, Portman RJ. Overweight, ethnicity, and the prevalence of hypertension in school-aged children. Pediatrics. 2004 Mar;113(3 Pt 1):475-82.

4. Ogden CL, Flegal KM, Carroll MD, Johnson CL. Prevalence and trends in overweight among US children and adolescents, 1999-2000. JAMA. 2002 Oct 9;288(14):1728-32

5. Lauer RM, Clarke WR. Childhood risk factors for high adult blood pressure: the Muscatine Study. Pediatrics. 1989 Oct;84(4):633-41.

6. Lu Q, Ma CM, Yin FZ, Liu BW, Lou DH, Liu XL. How to simplify the diagnostic criteria of hypertension in adolescents. J Hum Hypertens. 2011 Mar;25(3):15963. doi: 10.1038/jhh.2010.46. Epub 2010 Apr 29. 
7. Marras AR, Bassaroe PP, Ruscazio M: The prevalence of paediatric hypertension, emphasising the need to use specific population references: the Sardinian Hypertensive Addolecents Research Program Study. Cardiol Young 2009; 19:2333-2338.

8. Brady TM, Fivush B, Parekh RS, Flynn JT. Racial differences among children with primary hypertension. Pediatrics. $2010 \quad$ Nov;126(5):931-7. doi: 10.1542/peds.2009-2972. Epub 2010 Oct 18.
9. Ejike CE. Blood pressure to height ratios as simple, sensitive and specific diagnostic tools for adolescent (pre)hypertension in Nigeria. Ital J Pediatr. 2011 Jun 24;37:30. doi: 10.1186/1824-7288-37-30.

10. Galescu O, George M, Basetty S, Predescu I, Mongia A, Ten S, Bhangoo A. Blood Pressure over Height Ratios: Simple and Accurate Method of Detecting Elevated Blood Pressure in Children. Int J Pediatr. 2012;2012:253497. doi: 10.1155/2012/253497. Epub 2012 Apr 8.

\section{How to cite this article?}

Kurane AB, Kurane IA, N S Magdum, S Sumana. Study of blood pressure to height ratio and its relation with standard BP percentile charts to detect the prehypertension in age group 12 to 15 yrs. Pediatr Rev: Int J Pediatr Res 2015;2(4):120-124.doi:10.17511/ijpr.2015.i04.15 